\section{Hematopoietic stem cell loss and hematopoi- etic failure in severe aplastic anemia is driven by macrophages and aberrant podoplanin expression}

\author{
Amanda McCabe,,$^{1 *}$ Julianne N.P. Smith,,$^{1 * \star}$ Angelica Costello, ${ }^{1}$ \\ Jackson Maloney, ${ }^{1}$ Divya Katikaneni ${ }^{1}$ and Katherine C. MacNamara ${ }^{1}$
}

${ }^{1}$ Department for Immunology and Microbial Disease, Albany Medical College, NY, USA

AM and JNPS contributed equally to this work. ${ }^{\star}$ Current address: Boston Children's Hospital, Division of Hematology/Oncology, Harvard Medical School, Karp Family Research Lab, Boston, MA, USA **Current address: Case Western Reserve University, Department of Medicine, Wolstein Research Building, Cleveland, OH, USA

\title{
ABSTRACT
}

evere aplastic anemia (SAA) results from profound hematopoietic

S stem cell loss. T cells and interferon gamma (IFN $\gamma$ ) have long been associated with SAA, yet the underlying mechanisms driving hematopoietic stem cell loss remain unknown. Using a mouse model of SAA, we demonstrate that IFN $\gamma$-dependent hematopoietic stem cell loss required macrophages. IFN $\gamma$ was necessary for bone marrow macrophage persistence, despite loss of other myeloid cells and hematopoietic stem cells. Depleting macrophages or abrogating IFN $\gamma$ signaling specifically in macrophages did not impair T-cell activation or IFN $\gamma$ production in the bone marrow but rescued hematopoietic stem cells and reduced mortality. Thus, macrophages are not required for induction of IFN $\gamma$ in SAA and rather act as sensors of IFN $\gamma$. Macrophage depletion rescued thrombocytopenia, increased bone marrow megakaryocytes, preserved platelet-primed stem cells, and increased the platelet-repopulating capacity of transplanted hematopoietic stem cells. In addition to the hematopoietic effects, SAA induced loss of nonhematopoietic stromal populations, including podoplanin-positive stromal cells. However, a subset of podoplanin-positive macrophages was increased during disease, and blockade of podoplanin in mice was sufficient to rescue disease. Our data further our understanding of disease pathogenesis, demonstrating a novel role for macrophages as sensors of IFN $\gamma$, thus illustrating an important role for the microenvironment in the pathogenesis of SAA.

\section{Introduction}

Severe aplastic anemia (SAA) is a rare, lethal bone marrow (BM) failure disease that can be inherited or acquired. The most effective treatment for SAA is BM transplantation but disease management also includes immunosuppressive therapy (IST). Not all patients are good transplant candidates, however, and IST responsiveness varies. Therefore more specific treatments are necessary. ${ }^{1,2}$

Chemical-induced toxicity, myeloablation, and lymphocyte infusion-based BM destruction have been used to model SAA in mice and define factors critical for initiating disease. ${ }^{1,2}$ SAA can be acquired as a result of radiation, toxic drug exposure, or infection. Acquired forms are often immune-mediated, ${ }^{3}$ thus the lymphocyteinfusion model is clinically relevant. Sublethal irradiation and subsequent lymphocyte or bulk splenocyte transfer elicits pancytopenia and death within 2-3 weeks. ${ }^{4}$ Importantly, disease progression and IST treatment responses are similar to those in SAA patients. ${ }^{5} \mathrm{~T}$ cells promote hematopoietic stem cell (HSC) loss during SAA through a "bystander effect" involving inflammatory cytokines, including IFN $\gamma^{6.9}$ IFN $\gamma$ negatively regulates HSC function, and it was first observed over thirty years ago that SAA patients show elevated IFN $\gamma$ levels. ${ }^{10}$ Despite this knowledge, the
Haematologica 2018

Volume 103(9):1451-1461

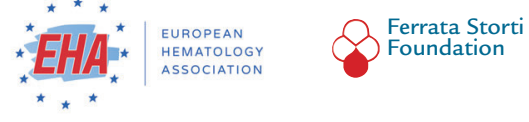

\section{Correspondence:}

macnamk@amc.edu

Received: January 30, 2018

Accepted: May 14, 2018.

Pre-published: May 17, 2018.

doi:10.3324/haematol.2018.189449

Check the online version for the most updated information on this article, online supplements, and information on authorship \& disclosures: www.haematologica.org/content/103/9/1451

(C)2018 Ferrata Storti Foundation

Material published in Haematologica is covered by copyright. All rights are reserved to the Ferrata Storti Foundation. Use of published material is allowed under the following terms and conditions:

https://creativecommons.org/licenses/by-nc/4.0/legalcode. Copies of published material are allowed for personal or internal use. Sharing published material for non-commercial purposes is subject to the following conditions:

https://creativecommons.org/licenses/by-nc/4.0/legalcode, sect. 3. Reproducing and sharing published material for commercial purposes is not allowed without permission in writing from the publisher. 
underlying mechanisms whereby IFN $\gamma$ drives SAA are still unknown.

Thrombocytopenia causes substantial morbidity and mortality in SAA patients. ${ }^{11}$ Megakaryocytes (Mks) not only produce platelets via thrombopoiesis, they serve as critical niches for HSCs. ${ }^{12,13}$ Thrombopoiesis is regulated by soluble factors, vascular integrity, and extracellular matrix composition, and requires adequate numbers and location of Mks. ${ }^{14}$ Platelet-biased HSCs, including HSCs that highly express CD41, have been observed in settings of inflammation and aging, where increased platelet output may be necessary to maintain vascular function. ${ }^{15,16}$ Inflammation can impact megakaryopoiesis and thrombopoiesis, though it is unclear whether this process is modulated in SAA.

Bone marrow macrophages (Mфs) support stromal niche cell function at steady-state, ${ }^{17-19}$ however, little is known about the impact of inflammation on BM Mфs. Herein, we

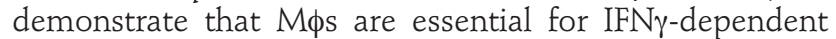

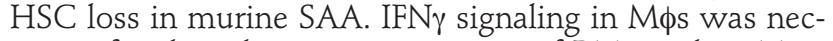
essary for the selective maintenance of $\mathrm{BM}$ resident $\mathrm{M \phi s}$, whereas all other myeloid cells were diminished. Targeting M $\phi$ s during SAA, via depletion or blocking their ability to respond to IFN $\gamma$, rescued HSCs and markedly improved survival. We demonstrate a key role for BM $M \phi s$ in sensing IFN $\gamma$, and our findings suggest that dysfunctional megakaryopoiesis and thrombopoiesis underlie hematopoietic collapse in SAA.

\section{Methods}

\section{Mice}

C57BL/6 $\left(\mathrm{H}^{\mathrm{b} / \mathrm{c}}\right)$ and BALB/c $\left(\mathrm{H}^{\mathrm{d} / \mathrm{d}}\right)$ mice were from Taconic (Albany, NY, USA). C57BL/6-TG(UBC-GFP)30Scha/J mice and ACTB-tdTomato mice were from Jackson Laboratory (Bar Harbor, ME, USA). MIIG (M $\phi$-insensitive to IFN $\gamma)$ mice $^{20}$ were a gift from Dr. Michael Jordan. Hybrid B6 F1 (Hb/d) were generated by crossing C57BL/6 with BALB/c mice. To generate MIIG F1 (Hb/d) mice, MIIG (C57BL/6 background) and BALB/c mice were crossed. Hybrid F1 progeny were screened by PCR to identify mice carrying the MIIG transgene, and MIIG-negative mice were included as littermate controls (LC). Mice were bred and housed in the Animal Research Facility at Albany Medical College (AMC) under microisolator conditions. Protocols were approved by the AMC Institutional Animal Care and Use Committee.

\section{SAA induction}

Age- (6-8 weeks) and sex-matched B6 F1 mice were sublethally irradiated (300 RADs) using a ${ }^{137} \mathrm{Cs}$ source four hours prior to intraperitoneal (i.p.) transfer of $5 \times 10^{7} \mathrm{C} 57 \mathrm{BL} / 6$ splenocytes. ${ }^{4}$ Mice were euthanized by $\mathrm{CO}_{2}$ inhalation at the indicated day post splenocyte transfer (d.p.s.t.). For survival studies, mice were examined twice daily and humanely euthanized upon $20 \%$ loss of initial body weight or if found moribund. Surviving mice were euthanized approximately ten days after the last mouse succumbed to disease.

\section{Cell preparation and flow cytometry}

Bone marrow was flushed from femurs and tibias, and spleens were homogenized. After red blood cell (RBC) lysis single-cell suspensions were plated and stained. Surface-stained cells underwent nuclear or cytoplasmic permeabilization (BD Pharmingen) prior to T-bet (4B10) and IFNy staining, respectively. Data were collected on an LSR II (BD Biosciences) and analyzed using FlowJo software (TreeStar, Ashland, OR, USA).

\section{Bone-associated cell analysis}

Bones were crushed using a mortar and pestle in HBSS, washed and twice digested in $2 \mathrm{mg} / \mathrm{mL}$ collagenase type 2 and $1 \mathrm{X}$ trypsin enzyme solution at $37^{\circ} \mathrm{C}$ (with rocking, $30 \mathrm{~min}$ ).

\section{Complete blood cell count}

Complete blood counts (CBCs) were determined using an automated hematology analyzer (Cell Dyn 3700, Abott Laboratories).

\section{Tissue preparation for protein quantification}

Bone marrow cell lysates were homogenized with a pestle in a buffer containing IGEPAL CA-630 and proteinase inhibitors for protein analysis.

\section{Transplantation}

150 HSCs ( $\left.\mathrm{Lin}^{-} \mathrm{cKit}^{+} \mathrm{CD} 48-\mathrm{CD} 150^{+}\right)$were sorted from PBS- and clod-lip-treated ACTB-tdTomato F1 mice 8 d.p.s.t. and transplanted separately into lethally irradiated F1 recipients with $2.5 \times 10^{5}$ protective F1 whole BM cells.

\section{Macrophage depletion and antagonist delivery}

$250 \mu \mathrm{L}$ of PBS- or clodronate-liposomes (ClodronateLiposomes. com) was administered intravenous (i.v.) at 1 d.p.s.t. (day 7 analysis) or 1 and 7 d.p.s.t. (day 15 analysis). Anti-podoplanin (PDPN) antibody (clone 8.1.1) or Syrian Hamster IgG (both from BioXcell) was administered i.v. 3, 7, and 10 d.p.s.t. at $125 \mu \mathrm{g} /$ dose.

\section{Platelet analysis}

Fluorescently-tagged anti-Gp1b $\beta$ antibodies (Emfret Analytics) were administered to mice 5 or 10 d.p.s.t., according to the manufacturer's instructions.

\section{Histology}

Sternums were fixed in $10 \%$ buffered formalin, decalcified in $14 \%$ EDTA, and paraffin-embedded. Megakaryocytes were stained with anti-rat GP1b $\beta$ (Emfret) and nuclear fast red (Poly Scientific R\&D) counterstaining. Images were taken on an Olympus SC30 light microscope using CellSens software.

\section{Statistical analysis}

Analysis was performed using Prism software. Two-tailed Student $t$-test was used to compare between indicated groups, unless otherwise reported.

\section{Results}

\section{BM macrophages are maintained during SAA}

Hematopoietic stem cell loss and BM destruction are key features of SAA and are associated with cytokine production by $T$ cells. ${ }^{6-8}$ It is unclear, however, if inflammation depletes HSCs directly or through the microenvironment. To examine resident M $\phi$ s in SAA, we used an established murine model of $\mathrm{BM}$ failure involving histocompatibility mismatched recipients and splenocyte infusion. ${ }^{4}$ SAA was induced via sublethal irradiation of F1 hybrids (C57BL/6 $\mathrm{x}$ $\mathrm{BALB} / \mathrm{c})$, followed by adoptive transfer of C57BL/6 splenocytes (Online Supplementary Figure S1A). Significant cytopenias were observed in SAA mice at 8 and 15 d.p.s.t. compared to radiation controls (Rad) (Online Supplementary Figure S1B-D). Thrombocytopenia was evident 8 d.p.s.t in control and SAA mice, and progressed in SAA mice (Online Supplementary Figure S1D). SAA-associated cytopenias coincided with BM hypocellularity (Figure 1A) and HSC loss (Online Supplementary Figure S1E-G). 


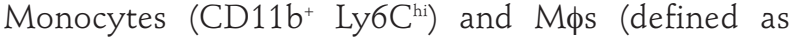

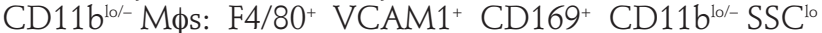

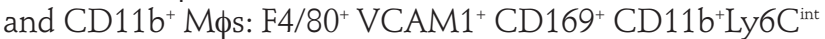
SSC $^{\text {lo; }}$ Online Supplementary Figure S2), were increased by frequency 8 d.p.s.t., relative to Rad mice (Figure 1C). At 15 d.p.s.t., CD11b+ Mps, monocytes, and neutrophils were reduced by frequency whereas $C D 11 b^{\text {lo/ }} M \phi$ frequencies were significantly increased (Figure 1D). Despite severe BM hypocellularity at 15 d.p.s.t., CD $11 b^{\text {loo- }} M \phi$ numbers remained stable (Figure $1 F)$. Thus, BM CD11b ${ }^{\text {lo/- }}$ Mфs per- sist despite SAA-associated cytopenias, myeloid cell loss, and HSC loss in SAA. These data suggest that CD11bo/ Mфs do not require hematopoietic input during SAA, potentially due to their long lifespan or ability to selfrenew.

IFN $\gamma$-dependent increase in BM macrophages during SAA drives HSC Ioss and thrombocytopenia

Interferon- $\gamma$ mediates SAA pathology ${ }^{5,9}$ and maintains BM M $\phi s$ during infection, ${ }^{21}$ thus we next addressed
A

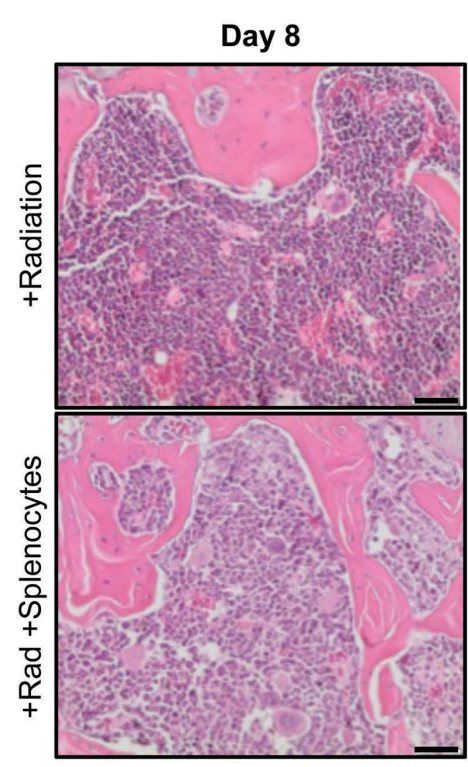

B

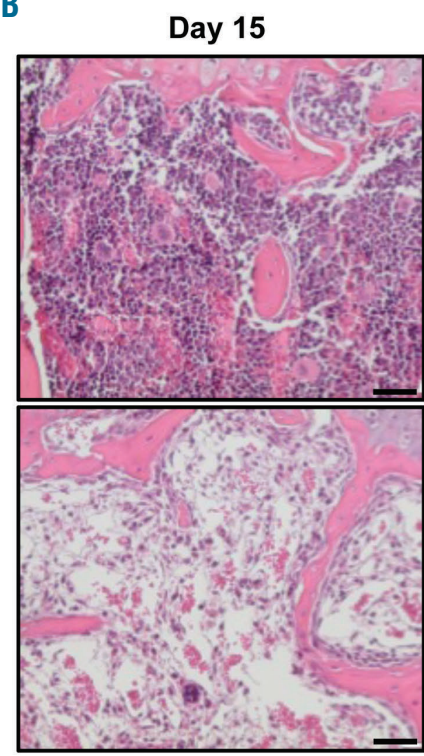

$\square+$ Rad.

+ Rad +Splen

C

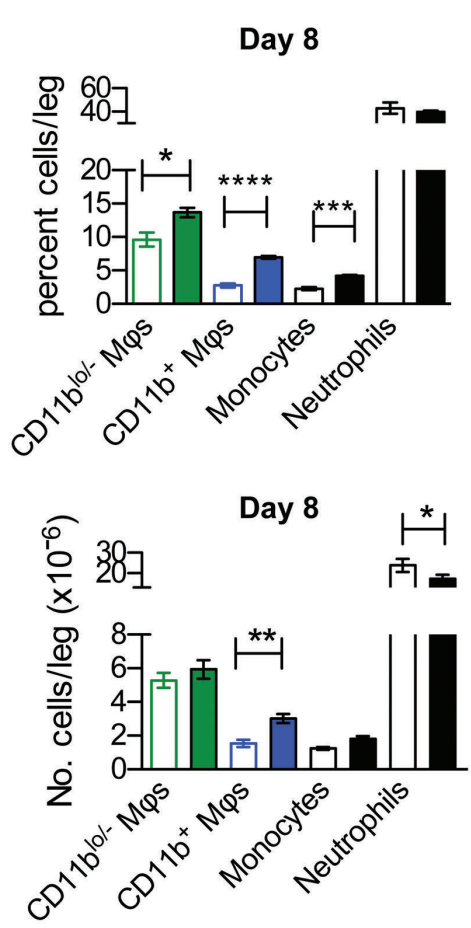

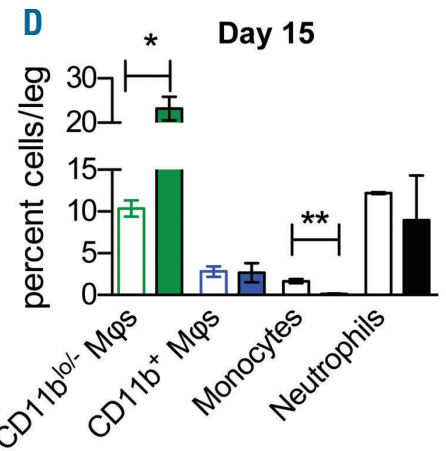

Day 15

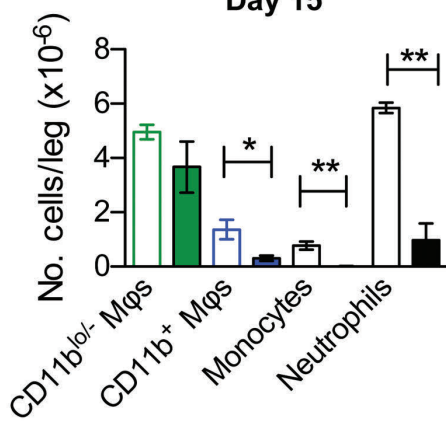

Figure. 1. Bone marrow (BM) macrophages are maintained during aplastic anemia. (A and B) Hematoxylin and eosin-stained $\mathrm{BM}$ in radiation control (Rad) (top) and severe aplastic anemia (SAA) (bottom) mice on days 8 and 15. Scale bar $=100 \mu m$. Frequencies $(C$ and $D)$ and numbers (E and $F$ ) of $C D 11 b^{1 / \%} M \phi s$, $\mathrm{CD} 11 \mathrm{~b}^{+} \mathrm{M} \phi \mathrm{s}$, monocytes, and neutrophils in radiation control (open bars) and SAA (filled bars) mice on days 8 and 15. Data represent one experiment repeated at least twice, $n=3-6$ mice/group. Mean \pm Standard Error of Mean is shown. $* P<0.05, * * P<0.01, * * * P<0.001$, $* * * * P<0.0001$ 
whether IFN $\gamma$ was necessary for preservation of M $\phi$ s during SAA. Using transgenic mice in which $M \phi$-lineage cells are insensitive to IFN $\gamma$ (referred to as MIIG mice) due to a CD68-driven dominant-negative IFN $\gamma$ receptor, ${ }^{20}$ we noted improved cellularity upon induction of SAA (Figure 2A and B). MIIG and littermate control (LC) mice showed no significant difference in response to sublethal radiation; however, CD11 $\mathrm{b}^{\mathrm{lo} /}$ and $\mathrm{CD} 11 \mathrm{~b}^{+} \mathrm{M} \phi \mathrm{s}$ were significantly reduced in MIIG mice relative to LC counterparts 8 d.p.s.t, when HSC loss is first noted (Figure 2C). Thus, we define a novel role for IFN $\gamma$ in maintaining and increasing $\mathrm{BM}$ Mфs during SAA.

Based on our prior findings in bacterial infection, where Mфs drive IFNY-induced HSC depletion, ${ }^{21}$ we predicted that the BM HSC pool would be preserved in MIIG mice during SAA. Indeed, HSCs were preserved in MIIG mice, relative to LCs (Figure $2 \mathrm{D}$ and $\mathrm{E}$ ), demonstrating IFN $\gamma$ sensing by $M \phi$-lineage cells drives HSC loss in SAA. Anemia was slightly, but significantly, ameliorated (Figure $2 \mathrm{~F}$ and $\mathrm{G}$ ) whereas thrombocytopenia was strikingly rescued in MIIG relative to LC controls (Figure 2H). In fact, platelet levels were higher in MIIG mice with SAA than in radiation-control mice. The robust platelet rescue suggested that IFN $\gamma$-stimulated M $\phi$ s contribute specifically to thrombocytopenia in SAA.

\section{MIIG mice exhibit increased CD41 ${ }^{\text {hi }}$ HSCs and megakaryocytes during SAA}

Inflammation-induced megakaryopoiesis reportedly relies on the emergence of a CD41 ${ }^{\text {hi }}$ stem-like Mk progenitor cell type (SL-MkP) within the phenotypic HSC gate. ${ }^{15}$ SL-MkPs self-renew and rapidly produce $\mathrm{Mks}$ and platelets, while CD41 $1^{\text {lo/int }} \mathrm{HSCs}$ contain multi-lineage potential. ${ }^{15,16}$ CD41 expression increased robustly on HSCs in MIIG relative to LC mice at day 15 p.s.t. (Figure $3 A)$, suggesting that IFN $\gamma$-sensing M $\phi s$ limit $C D 41^{\text {hi }} \mathrm{HSC}$ emergence in response to SAA-induced inflammation. MIIG and LC radiation-control mice exhibited similar numbers of $C D 41^{\text {lo/int }}$ and $C D 41^{\text {hi }}$ at days 8 and 15 p.s.t. (Figure $3 \mathrm{~B}$ ). In SAA conditions, however, MIIG mice exhibited significantly more CD41 ${ }^{\text {hi }}$ HSCs (Figure 3C), and increased CD41 $1^{\text {loint }}$ on day 15 p.s.t. than LC. Consistent with increased phenotypic SL-MkPs we observed increased BM Mks in MIIG SAA mice, relative to LC (Figure $3 \mathrm{D}$ and $\mathrm{E}$ ). Our data indicate that IFN $\gamma$ signaling in Mps during SAA is associated with rapid loss of both CD41 $1^{\text {lo/int }}$ and CD41 ${ }^{\text {hi }}$ HSCs, which correlates with Mk depletion and severe thrombocytopenia. Moreover, SAAinduced mortality was significantly reduced in MIIG compared to LC mice (Figure 3F). Thus, M $\phi$ s are key sensors of IFN $\gamma$, and our data strongly suggest that M $\phi$ s drive disease and death by reducing platelet-biased CD $41{ }^{\text {hi }} \mathrm{HSCs}$.
A
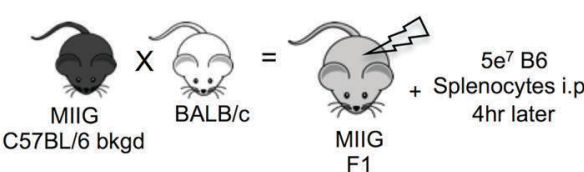

C
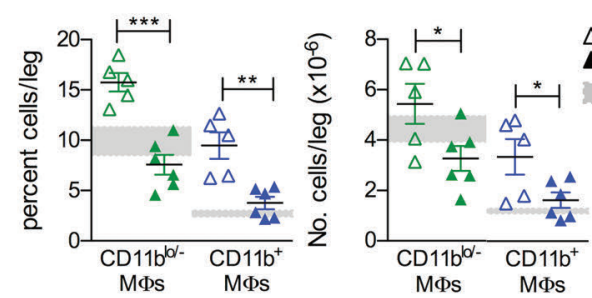

F

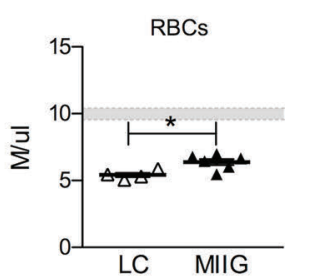

G

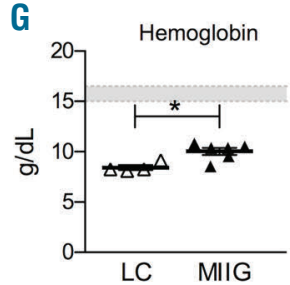

B

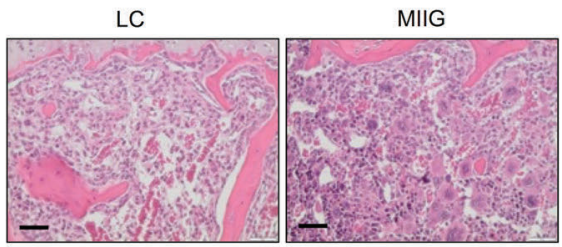

D

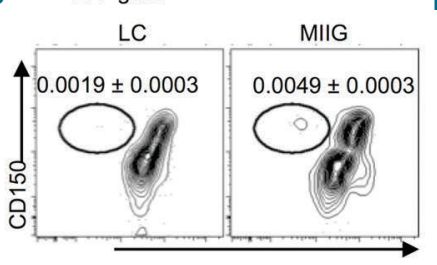

$\mathrm{E}$

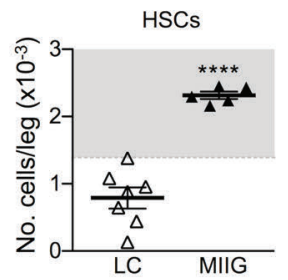

H

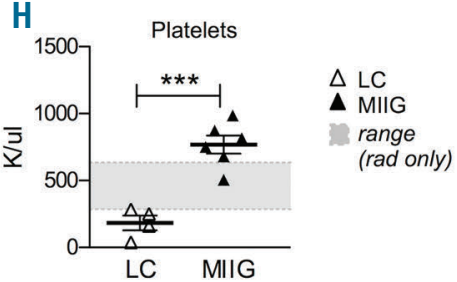

Figure 2. IFN $\gamma$ sensing by macrophages is required for bone marrow (BM) macrophage maintenance and hematopoietic stem cell (HSC) loss in aplastic anemia. (A) Severe aplastic anemia (SAA) was induced in MIIG and littermate control (LC) F1 hybrids. (B) Hematoxylin and eosin-stained BM of LC and MIIG mice 15 days

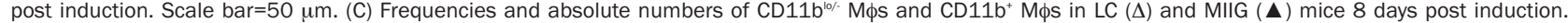
Shading represents ranges of each M $\phi$ population in radiation control LC and MIIG mice. (D) CD150 and CD48 expression on BM Lin- c-Kit ${ }^{+}$(LK ${ }^{+}$) cells. Numbers represent mean $\mathrm{HSC}\left(\mathrm{LK}^{+} \mathrm{CD} 150^{+} \mathrm{CD} 48^{-}\right)$frequency \pm Standard Error of Mean (SEM). (E) HSC numbers in MIIG $(\Delta)$ and $\mathrm{LC}(\boldsymbol{\Delta})$ mice 8 days post induction. Shading represents ranges of radiation control LC and MIIG mice. (F) Red blood cells (RBCs), (F) hemoglobin, and (H) platelets in the blood 15 days post induction. Shading represents ranges of radiation control LC and MIIG mice. Data represent one experiment repeated at least two times, $\mathrm{n}=5-7 \mathrm{mice} / \mathrm{group}$. Mean $\pm \mathrm{SEM}$ is shown. $* P<0.05, * * P<0.01, * * * P<0.001, * * * * P<0.0001$. 
Clodronate-liposomes specifically deplete macrophages, increase CD41 ${ }^{\mathrm{h}} \mathrm{HSCs}$ and platelets, and rescue survival during SAA

To test the impact of $M \phi$ depletion on SAA pathogenesis, we administered clodronate-encapsulated liposomes (clod-lip) to mice one day after SAA induction. BM M $\phi s$ were significantly and specifically reduced 8 d.p.s.t. (Figure 4A). Monocytes and neutrophils are also phagocytic and may be transiently depleted; however, they were quickly replaced and no sustained depletion was observed with clod-lip. M $\phi$ depletion correlated with improved cellularity at day 15 (Figure $4 \mathrm{~B}$ and C), increased total HSCs (Online Supplementary Figure S3A), and increased CD41 ${ }^{\mathrm{hi}}$ HSCs at 8 and 15 p.s.t (Figure 4D), thus supporting the idea that Mфs negatively regulate HSCs during SAA. Macrophage-colony stimulating factor (M-CSF) is critical for tissue $\mathrm{M} \phi$ survival and self-renewal, ${ }^{22,23}$ and similar to clod-lip administration, M-CSFR antagonism significantly increased HSCs during SAA (Online Supplementary Figure $S 3 B$ ). Similar to MIIG SAA mice, CD $41^{\text {hi }}$ HSCs correlated with increased circulating platelets, significantly increased BM Mks, and improved survival (Figure 4E-G). HSCs were increased in both MIIG SAA and M $\phi$-depleted SAA mice, while more downstream progenitors, including shortterm HSCs and multipotent progenitors (MPPs), were more variable (Online Supplementary Figure S4A). Consistent with improved thrombocytopenia in both models, a similar and significant increase in megakaryocyte progenitors was observed in MIIG SAA mice and M申-depleted SAA mice (Online Supplementary Figure S4B).

A similar rate of platelet removal from circulation was observed in PBS- and clod-lip-treated SAA mice (Figure 4H and I). Thus, improved platelet counts were not due to loss of consumption by Mфs. The increase in BM Mks and significantly reduced mortality in M $\phi$-depleted mice compared to PBS-lip-treated controls demonstrate M $\phi$ s drive SAA mortality, possibly via their ability to restrict phenotypically-defined platelet-biased HSCs. Thus, HSC loss and thrombocytopenia is dependent on $M \phi s$ and $M \phi$ growth factors in SAA.

\section{T-cell responses are not impaired in clod-lip-treated and MIIG mice}

Macrophages may drive HSC loss by enhancing activated T-cell infiltration into the BM; therefore, we tracked donor $\mathrm{T}$ cells by inducing SAA with splenocytes from UBC-GFP mice (C57BL/6 background). Expression of the T-helper 1 transcription factor T-bet, which is expressed in $\mathrm{T}$ cells of SAA patients and increases IFN $\gamma$ gene transcription, ${ }^{7}$ was not diminished in T cells from M $M$-depleted

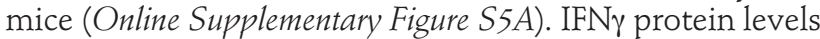
(Online Supplementary Figure S5B) and IFN $\gamma$-secreting donor T cells (Online Supplementary Figure S5C-E) in the $\mathrm{BM}$ of SAA mice were also unaffected by $\mathrm{M} \phi$ depletion. $\mathrm{T}$ -
A

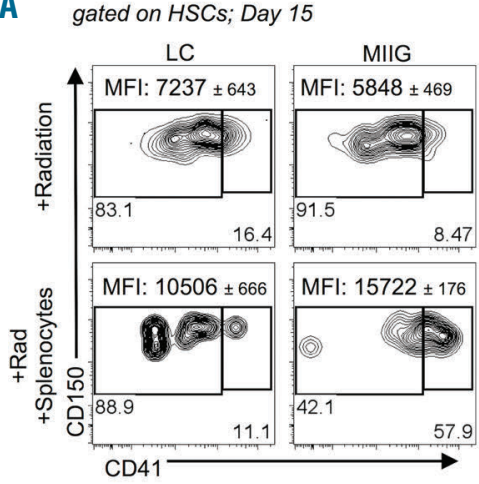

D

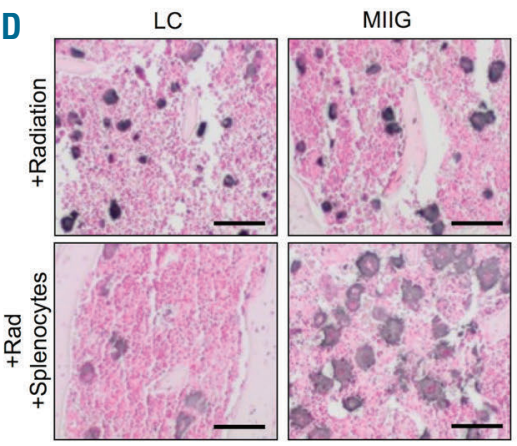

B

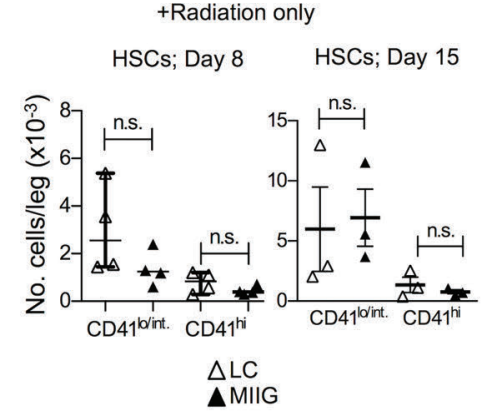

E

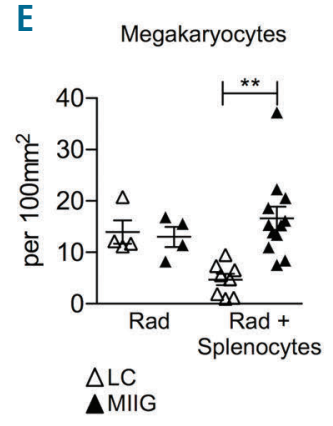

F
C

\section{+Rad +Splenocytes (SAA)}

HSCs; Day 8 HSCs; Day 15
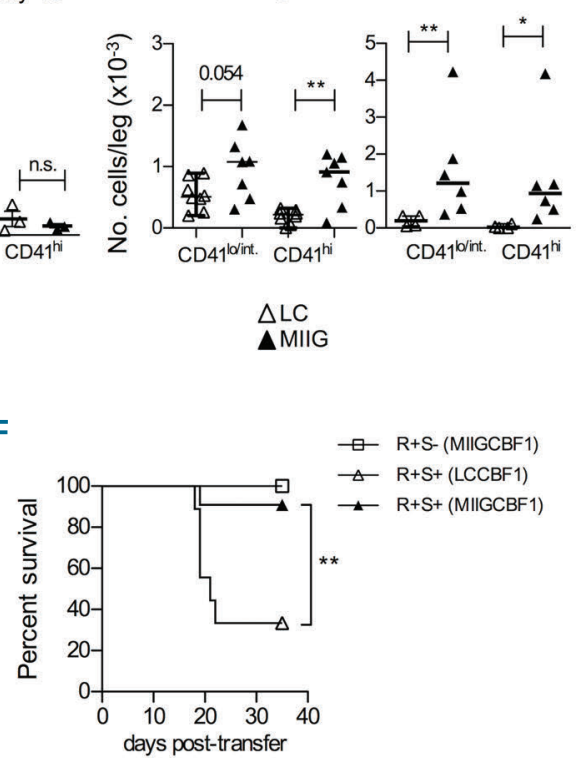

Figure 3. MIIG mice exhibit increased CD41 hi hematopoietic stem cells (HSC), increased megakaryocytes in the bone marrow (BM), and reduced mortality during aplastic anemia. (A) CD41 expression on BM HSCs in radiation control (Rad) (top) and severe aplastic anemia (SAA) (bottom) MIIG and LC mice 15 days post-spleno-

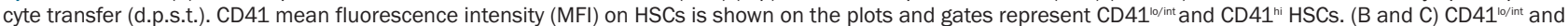
CD41 ${ }^{\text {hi }}$ HSC numbers in MIIG $(\boldsymbol{\Delta})$ versus LC $(\Delta)$ mice on days 8 (mean is shown) and 15 (median is shown, and a Mann-Whitney test was used to compare between groups). $* P<0.05, * * P<0.01$. (D) Gp1b $\beta$ staining in BM of Rad and SAA MIIG ( $\mathbf{\Delta}$ ) and LC (r) mice 15 d.p.s.t. Scale bar $=100 \mu \mathrm{m}$. (E) GP1b $\beta^{+}$megakaryocytes per 100 $\mathrm{mm}^{2}$ of sternal BM. Mean \pm Standard Error of Mean (SEM) is shown. $* * P<0.01$. (F) Kaplan-Meier survival curve for radiation control (Rad; LC and MIIG mice; $\square$, $\mathrm{n}=4$ ) and SAA MIIG $(\boldsymbol{\Delta} ; \mathrm{n}=9)$ and LC $(\Delta ; \mathrm{n}=11)$ mice. Log-rank (Mantel-Cox) test was used to compare between groups. ${ }^{*} P<0.05$. 
bet $^{+}$donor T-cell numbers, IFN $\gamma$ protein levels, and IFN $\gamma^{+}$ donor T cells were also comparable between LC and MIIG mice in SAA (Online Supplementary Figure S5F-J). Though donor $\mathrm{T}$ lymphocytes are necessary for disease initiation, HSC loss during SAA occurred independently of the direct effects of T-cell-derived IFN $\gamma$. Rather, HSC loss occurred through $M \phi$-dependent sensing of T-cell-derived IFN $\gamma$.

Interferon- $\gamma$ acts on M $\phi s$ to promote inflammation during disease contributing to $M 1$ polarization of $M \phi s^{24,25}$ and inflammation can impact HSC pool size and function. ${ }^{26,27}$ However, we observed similar levels of inflammatory factors previously associated with SAA, including TNFo, IL-6, and IL-1 $\beta$, in the BM of MIIG and LC mice during SAA (Online Supplementary Figure S6A). Furthermore, M $\phi$ depletion did not alter TNF $\alpha$, IL-6, and IL-1 $\beta$ levels (Online Supplementary Figure S6B), demonstrating that HSC loss is not due to induction of a broad inflammatory response during SAA. Despite the similar inflammatory milieu, purified M $\phi s$ from LC and MIIG SAA mice exhibited differential expression of genes associated with $\mathrm{M} 1$ and $\mathrm{M} 2$ polarization, programs associated with inflammatory and wound healing, respectively. ${ }^{28}$ In MIIG-derived CD11b ${ }^{+}$
Mфs, we observed a reduction in inducible nitric oxide synthesis (Nos2) expression, whereas induction of arginase 1 was seen in CD11bo/ M S6C). Elevated Nos2 expression and increased nitric oxide concentrations are associated with disease in SAA patients ${ }^{29}$ thus IFN $\gamma$ signaling in M $\phi$ s may drive pathological M1 polarization in SAA.

\section{M $\phi$ depletion increases functional platelet-biased HSCs}

To examine $M \phi$-dependent regulation of HSC function and lineage bias in SAA, we transplanted HSCs sorted from the BM of PBS- or clod-lip-treated SAA mice 8 d.p.s.t. (Figure 5A). To our knowledge, HSC function and lineage bias have not previously been assayed in models of SAA, likely due to the severity of BM hypocellularity. HSCs exposed to M $\phi s$ during SAA showed little repopulating activity, indicating that exposure to secondary stress severely compromised their function, whereas HSCs from clod-lip-treated SAA mice exhibited platelet-, myeloid-, and lymphoid-repopulating capacity (Figure 5B). Thus our data demonstrate that in SAA, M $\phi$ s reduce HSC function and impair platelet output.
A

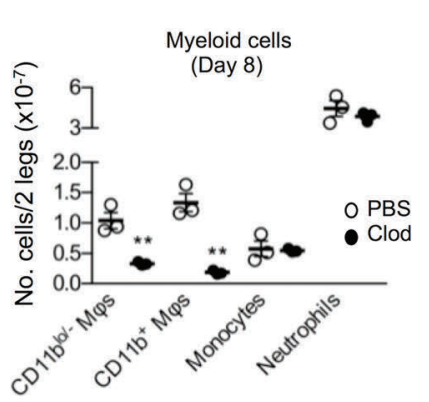

D

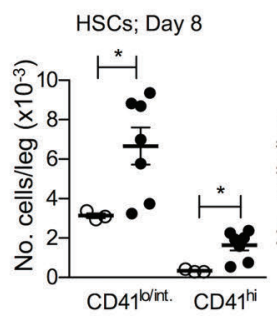

B

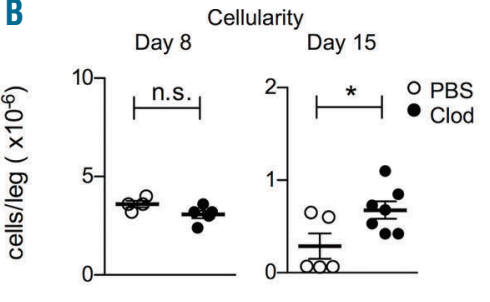

C

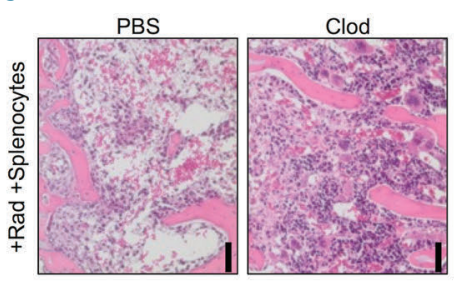

E
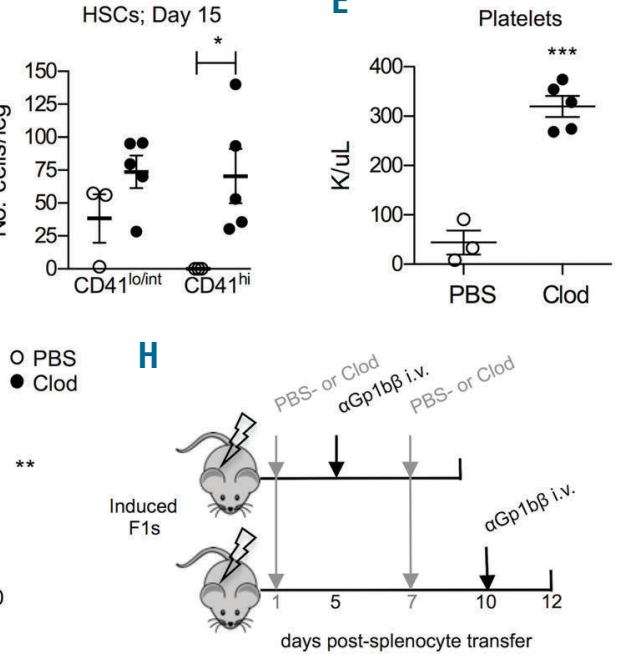

F
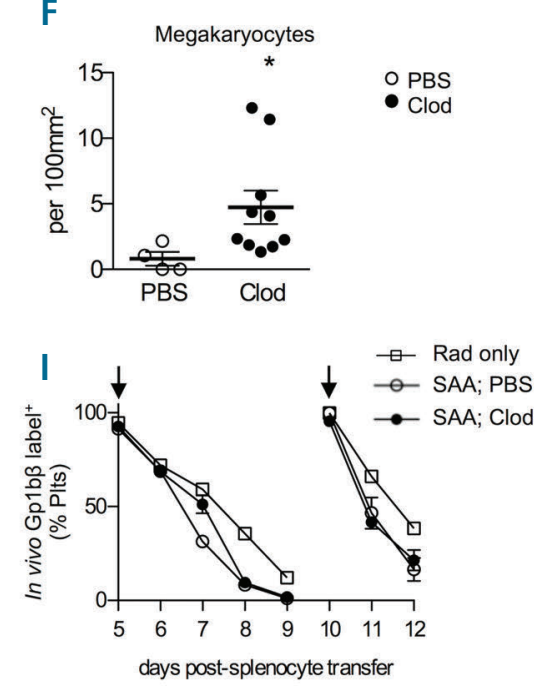

Figure 4. Clodronate-liposomes specifically deplete macrophages and rescue hematopoietic stem cell numbers during aplastic anemia. (A) Myeloid bone marrow $(B M)$ cell numbers in PBS- $(O)$ or clodronate-loaded (Clod; $\bullet$ ) liposome-treated severe aplastic anemia (SAA) mice. (B) BM cellularity in PBS- (O) or clodronate-loaded (Clod; •) liposome-treated SAA mice on days 8 and 15 post-splenocyte transfer (p.s.t.) is shown. (C) Hematoxylin and eosin-stained BM from PBS- or clod-lip-treated radiation control (top) and SAA (bottom) mice 15 days post splenocyte transfer (d.p.s.t.). Scale bar $=50 \mu \mathrm{m}$. (D) CD41//int and CD41 $\mathrm{HSC}$ numbers 8 and 15 days d.p.s.t. $* P<0.05$. (E) Platelets in the blood 15 d.p.s.t. $* * * P<0.001$. (F) GP1b $\beta^{+}$megakaryocytes per $100 \mathrm{~mm}^{2}$ of BM 15 d.p.s.t. Mean \pm Standard Error of Mean is shown. ${ }^{*} P<0.05$. (G) Kaplan-Meier survival curve for mice with SAA treated with PBS- $(0 ; n=8)$ or clod-lip $(\bullet ; n=9) 1$ and 7 d.p.s.t. Log-rank (Mantel-Cox) test was used to compare between groups. $* * P<0.01$. (H) Schematic showing administration of anti-Gp1b $\beta$ antibodies to mice 5 or 10 d.p.s.t. (I) Labeled platelets were measured in the blood over time. Two-way ANOVA was used to compare between groups. $P<0.0001$ and $P=0.0007$ for SAA-PBS versus radiation control (Rad) at days 5-9 p.s.t. and days 10-12 p.s.t., respectively; $P<0.0001$ for SAA-Clod versus Rad at days 5-9 p.s.t. and days $10-12$ p.s.t.; $P=0.004$ and $P=0.7$ for SAA-PBS versus SAA-Clod at days 5-9 p.s.t. and days 10-12 p.s.t., respectively. 
Aberrant podoplanin expression during SAA drives HSC loss, thrombocytopenia, and death

Macrophages negatively regulated both HSCs and Mks, and we questioned whether M申s also regulate nonhematopoietic BM stromal cells, known to be impaired in SAA patients. ${ }^{30-32}$ In contrast to radiation alone, SAA reduced osteoblastic and endothelial cells (Online Supplementary Figure S7A and B). Because SAA was associated with severe thrombocytopenia we examined expression of podoplanin (PDPN), recently identified in the BM and shown to increase platelet production. ${ }^{33}$ We noted a striking loss in $\mathrm{PDPN}^{+}$stromal cells in SAA (Online Supplementary Figure S7C and D). At the same time, however, we observed induction of PDPN on hematopoietic

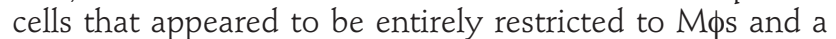
majority were $\mathrm{CD} 11 \mathrm{~b}^{\mathrm{lo}-} \mathrm{M} \phi \mathrm{s}$ (Figure 6A and $\mathrm{B}$, and Online Supplementary Figure S8A). We found no change in PDPN expression among other hematopoietic or nonhematopoietic stromal cells (data not shown). In addition, we observed increased podoplanin (gp38) transcripts specifically in the CD11 $b^{10 /-} \mathrm{M} \phi s$, relative to CD $11 b^{+} \mathrm{M} \phi s$, $\mathrm{T}$ cells, and neutrophils in SAA mice (Figure 6C). PDPN ${ }^{+}$ Mфs were reduced in MIIG mice relative to controls, though PDPN MFI was unchanged (Online Supplementary
Figure $S 8 B$ and $C)$. This suggests that IFN $\gamma$ increased numbers of $\mathrm{PDPN}^{+} \mathrm{BM} \mathrm{M} \phi \mathrm{s}$, rather than directly regulating PDPN expression, during SAA.

To determine if aberrant PDPN expression on M $\phi s$ mediated pathology during SAA, we administered an antiPDPN monoclonal antibody during SAA. PDPN blockade significantly increased CD41/ $1^{\text {lint }}$ and CD41 $1^{\text {hi }} \mathrm{HSCs}$ and resulted in a preservation of $\mathrm{BM}$ cellularity compared to isotype control treatment (Figure 7A and B). Administration of an anti-PDPN antibody did not rescue HSCs by reducing or impairing T-cell activation because similar numbers of $\mathrm{T}^{-}$bet $^{+} \mathrm{CD} 4$ and CD8 $\mathrm{T}$ cells and IFNy levels were observed in the BM of anti-PDPN and controltreated mice during SAA (Online Supplementary Figure S9A and $B$ ). Consistent with improved HSC numbers PDPN blockade rescued thrombocytopenia, increased BM Mks, and increased survival in SAA (Figure 7C-E). Anti-PDPN antibody conferred significant protection, whereas isotype-control antibody-treated mice had a median survival of only 16.5 days and died between days 12 and 19 induction of SAA.

PDPN-CLEC-2 interaction was reported to induce RANTES ${ }^{33}$ which can support platelet production, thus our finding that PDPN blockade improved thrombocy-
A<smiles>CCCC(=O)C12CC3CC(CC(C3)C1)C2</smiles>

induced

TdTomato F1

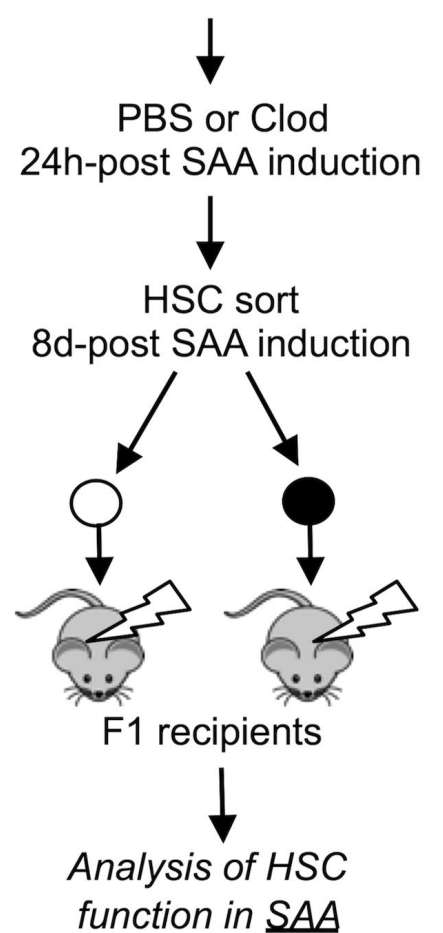

Analysis of HSC function in $\underline{S A A}$

B
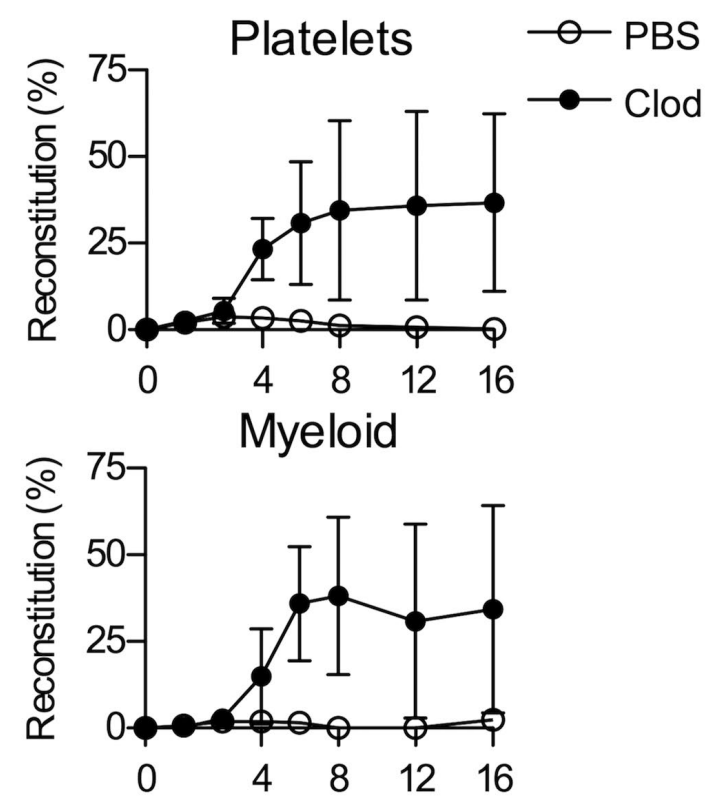

Lymphoid

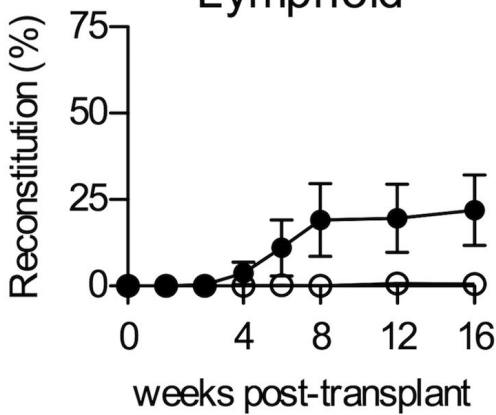

Figure 5. Macrophage depletion increases plateletbiased hematopoietic stem cells (HSC) in severe aplastic anemia (SAA). (A) HSC function in SAA was assessed by transplantation of HSCs from PBS- $\left({ }^{\mathrm{TM}}\right)$ or clod-lip (Clod; )-treated TdTomato+ F1 mice 8 days post-splenocyte transfer (d.p.s.t.). (B) Peripheral blood was analyzed for reconstitution at indicated time points. $P<0.0001$ for platelets, $P=0.002$ for myeloid, $P=0.06$ for lymphoid. Each transplantation data set represents one experiment, $n=4-5$ recipient mice per group. Two-way ANOVA was used to compare between groups. 

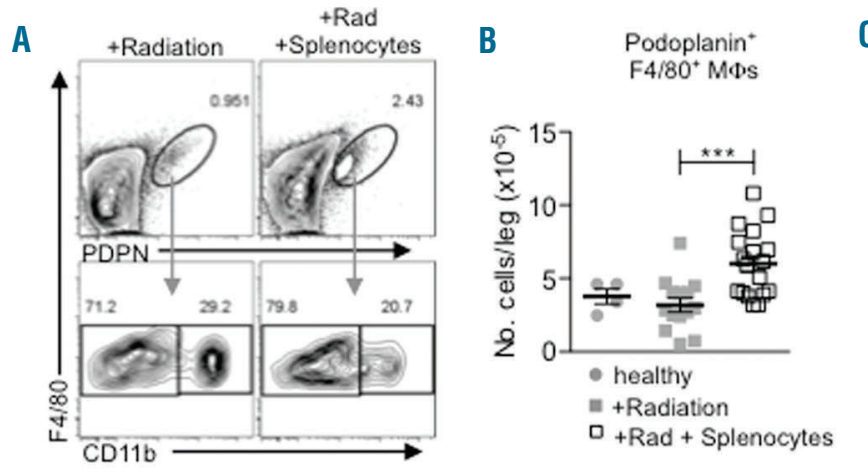

C

Gp38

Figure 6. Macrophages exhibit increased expression of podoplanin (PDPN) during severe aplastic anemia (SAA). (A) PDPN expression in bone marrow (BM) cells (top) 8 days post-splenocyte transfer (d.p.s.t.). CD11b expression among PDPN ${ }^{+} \mathrm{F} 4 / 80^{+}$cells (bottom). (B) PDPN ${ }^{+} \mathrm{F} 4 / 80^{+} \mathrm{M} \Phi$ numbers in healthy ( $\bullet$ ), radiation control ( $\square$ ), and SAA (+Rad +Splenocytes; $\square$ ) mice 8 d.p.s.t. (C) Gp38 expression in sort-purified BM populations, relative to $\beta$-actin and normalized to expression in neutrophils. Data represent data pooled from 3 independent experiments $n=5-10$ mice/group.

topenia was somewhat surprising. Consistent with a role for PDPN in RANTES production we observed increased RANTES during SAA (Online Supplementary Figure S9C), where we also observe increased $\mathrm{PDPN}^{+} \mathrm{M \phi s}$. PDPN blockade did not impact BM RANTES in SAA, likely because the antibody clone (8.1.1) does not interfere with CLEC-2 binding in vivo or in vitro (Online Supplementary Figure S9D-F)..$^{34}$ Thus, PDPN-dependent HSC loss and hematopoietic failure occurs via a RANTES-independent mechanism.

Administration of anti-PDPN antibody induced a specific decrease in CD11b $b^{\mathrm{lo}-}$ M $\phi$ s whereas CD11 ${ }^{\mathrm{b}+} \mathrm{M} \phi$ numbers were not significantly different (Figure 7F). This suggests PDPN signaling may be important for CD11b/ $\mathrm{M \phi}$ survival during SAA. It also demonstrates that selective reduction of $\mathrm{CD} 11 \mathrm{~b}^{\mathrm{lo} /} \mathrm{M} \phi \mathrm{s}$ is associated with improved survival during SAA. PDPN can bind and activate ezrin, radixin, and moesin family proteins to promote cytoskeletal reorganization and contractility of fibroblastic reticular cells in lymph nodes. ${ }^{34}$ Microenvironmental stiffness can reduce physical support for $\mathrm{HSCs}$ and $\mathrm{Mks},{ }^{35}$ and we observed reduced expression of $\alpha$-smooth muscle actin ( $\alpha$ SMA), a marker of contractile stress fibers, ${ }^{36}$ by $\mathrm{PDPN}^{+}$ BM Mфs at day 8 p.s.t. upon anti-PDPN treatment (Online Supplementary Figure S1OA and B). We also noted a striking increase in expression of arginase-1, a marker of M2-polar-

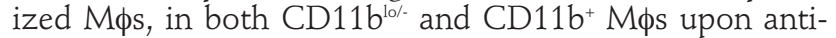
PDPN treatment (Online Supplementary Figure S10C). Thus, CD11 $b^{\text {lo/- }}$ M $\phi s$ aberrantly express PDPN in the BM during SAA, correlating with hematopoietic failure. Future studies are warranted to determine the precise impact of PDPN-expressing M $\phi$ s on the microenvironment and whether stiffness and $M \phi$-activation state contribute to SAA pathology.

\section{Discussion}

Hematopoietic stem cell loss and BM destruction are key features of SAA, and are associated with cytokine production by $T$ cells. ${ }^{6-8}$ It is still unclear, however, if inflammation depletes HSCs directly or does so through the microenvironment. Findings from SAA patient BM suggest that stromal support of hematopoietic cells is signifi- cantly reduced. . $^{30-32,37,38}$ In a mouse model of SAA, we observed reduced stromal cells, but, at the same time, BM

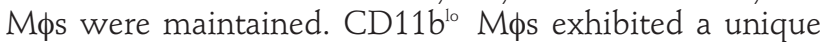
survival advantage in SAA that correlated with their expression of PDPN. Consistent with our findings, SAA patient BM exhibited CD169+ Mфs persistence despite significant reductions in nearly all other hematopoietic cell types. $^{31}$ Our findings reveal that, rather than direct IFN $\gamma$ mediated HSC depletion, IFN $\gamma$ signaling in M $\phi$ s promotes HSC loss during SAA. IFN $\gamma$ and M $\phi s$ limit CD41 ${ }^{\text {hi }} \mathrm{HSCs}$ during disease, thus contributing to severe thrombocytopenia and mortality in SAA (Figure 7G). To the best of our knowledge, this is the first in vivo study addressing the mechanistic role of the BM microenvironment in HSC loss and disease progression during SAA.

HSCs reportedly undergo apoptosis during SAA, ${ }^{39}$ yet studies in models of infection suggest that excessive differentiation and reduced self-renewal contribute to IFN $\gamma$ dependent HSC depletion..$^{40,41}$ We previously identified an IFN $\gamma$-dependent increase in monopoiesis during Ehrlichia muris infection, which occurred at the expense of HSCs. ${ }^{42}$ ${ }^{44}$ Monocytes are increased early in SAA, prior to their ultimate loss, supporting the idea that increased IFN $\gamma$-driven HSC differentiation contributes to HSC loss. It is also possible that increased apoptosis in SAA is a product of enhanced differentiating divisions that render HSCs more susceptible to inflammatory stress and/or cell death.

Aberrant immune cell function, specifically $\mathrm{T}$-cell activation and homing to the BM, is associated with SAA. ${ }^{7}$ Since IFN $\gamma$ primes M $\phi$ s for activation, ${ }^{25}$ and $M \phi s$ produce cytokines and present antigen to T cells, we predicted that IFN $\gamma$ signaling in M $\phi$ s increase T-cell activation. SAA progression is mitigated in MIIG mice, however, despite similar numbers of activated and IFN $\gamma$-secreting donor $\mathrm{T}$ cells in the BM. Cytokines associated with SAA (TNF $\alpha$, IL-1 $\beta$, and IFN $\gamma$ ) were also similarly induced. Thus, resident M $\phi s$ do not appear to drive disease through their capacity to present antigen to $\mathrm{T}$ cells or general inflammatory disposition. $M \phi$ polarization can contribute to disease through exaggerated inflammation and wound healing responses. ${ }^{28}$ During SAA, differential expression of M1-associated Nos2 was observed between the MIIG model and antiPDPN treatment and between $\mathrm{CD}_{11} \mathrm{~b}^{+}$and $\mathrm{CD} 11 \mathrm{~b}^{\mathrm{lo} /} \mathrm{M} \phi \mathrm{s}$, indicating functional differences between these two $M \phi$ 
A
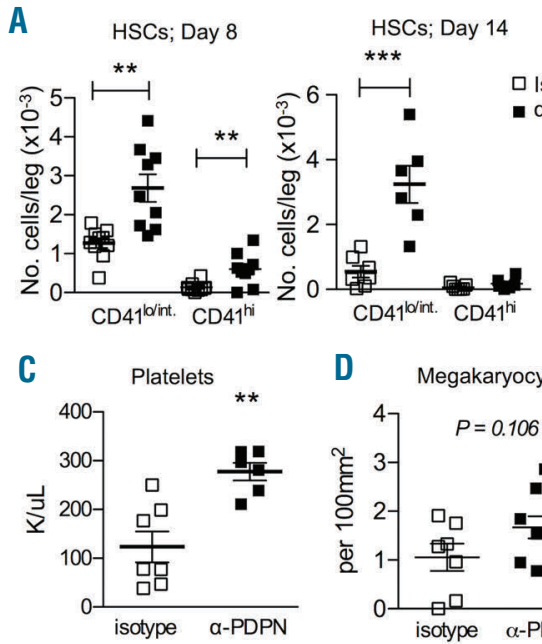

D

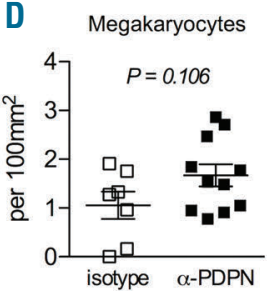

B

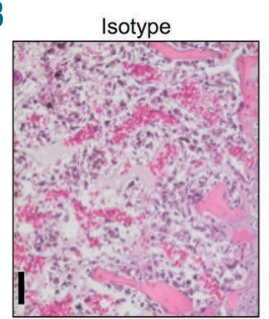

E

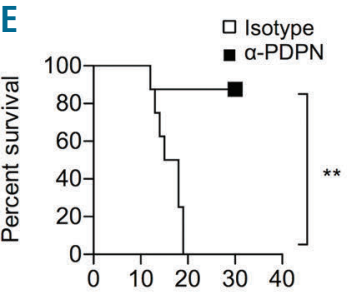

days post-transfer
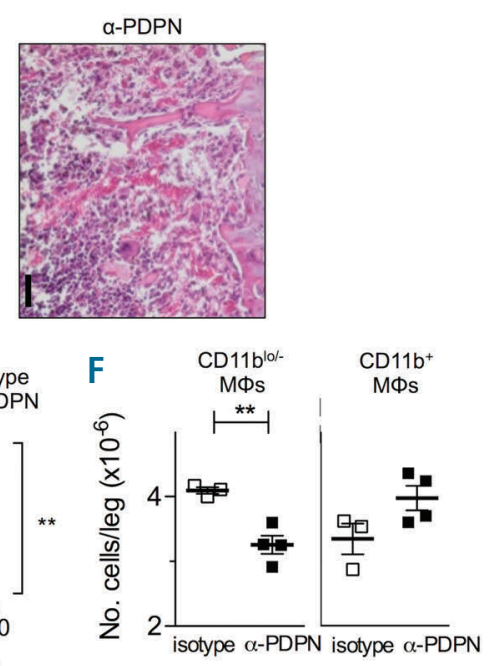

G

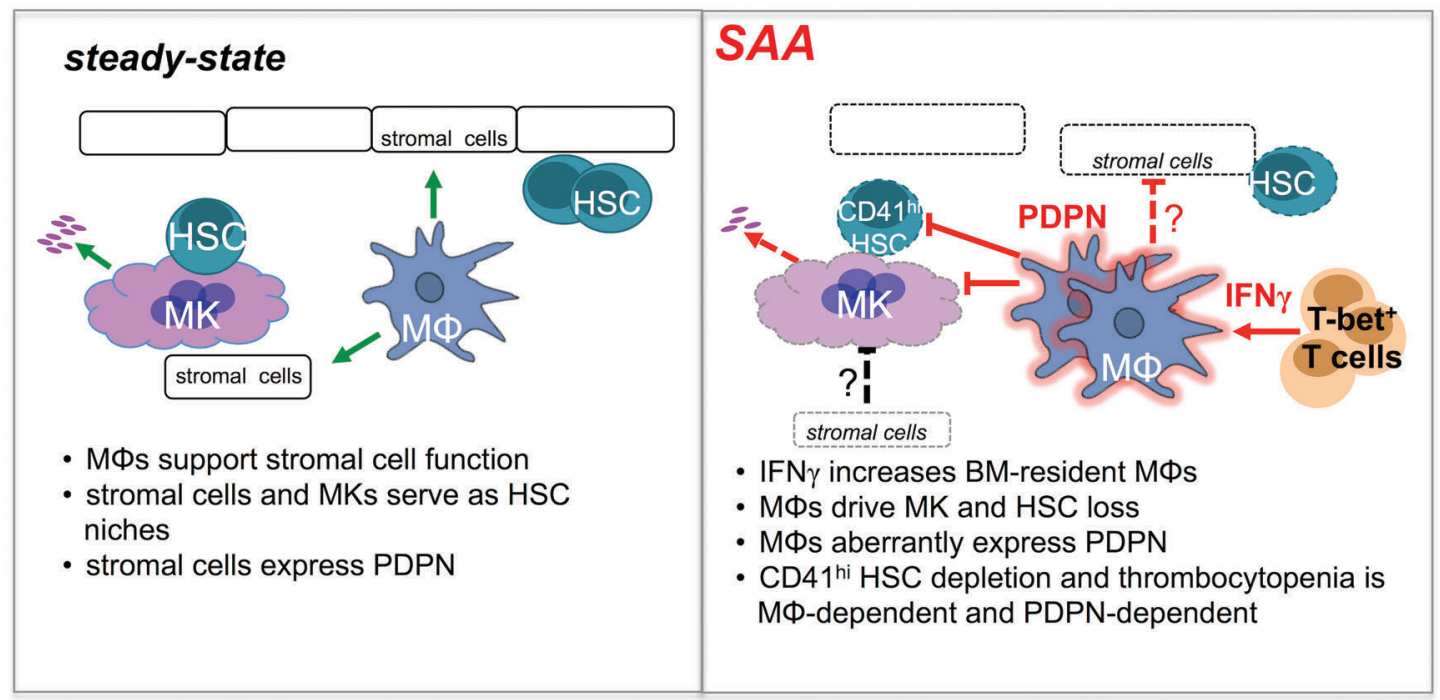

Figure 7. Podoplanin (PDPN) antagonism rescues hematopoietic stem cells (HSC), circulating platelet levels, and mortality. (A) CD41 ${ }^{\text {lo/int. }}$ and CD41 ${ }^{\text {hi }}$ HSC numbers in anti-PDPN ( $\square ; \alpha$-PDPN) or isotype control ( $\square$ )-treated mice 8 and 14 days post-splenocyte transfer (d.p.s.t.). (B) Hematoxylin and eosin-stained bone marrow (BM) from isotype control- or $\alpha$-PDPN-treated mice 14 d.p.s.t. Scale bar=50 $\mu \mathrm{m}$. (C) Platelets in the blood of isotype control- ( $\square$ ) and $\alpha$-PDPN ( $\square$ )-treated mice 14 d.p.s.t. (D) GP1b $\beta^{+}$megakaryocytes per $100 \mathrm{~mm}^{2}$ of BM 14 d.p.s.t. Data represent one experiment repeated at least twice, $\mathrm{n}=4-18 \mathrm{mice} / \mathrm{group}$. Mean \pm Standard Error of Mean is shown. $* * P<0.01, * * * P<0.001$. (E) Kaplan-Meier survival curve for $\alpha$-PDPN- ( $\square$ ) or isotype control ( $\square$ )-treated severe aplastic anemia (SAA) mice. Data represent one experiment, $n=8$ mice/group. Log-rank (Mantel-Cox) test was used to compare between groups. (F) CD11 $b^{1 \%}$ and $C D 11 b^{+} M \Phi$ populations were enumerated 8 d.p.s.t. (G) Schematic summarizing the steady state role(s) for MФs in the BM (left) and the impact of IFN $\gamma$ on the BM microenvironment and resulting HSC loss in SAA (right): increased PDPN-expressing MФs that drive reduced Mks, impaired platelet production, and correlate with reduced stromal.

populations. Of note, we observed increased expression of M2-associated arginase 1 in CD11b $b^{\text {lo/ }}$ M $\phi$ s from MIIG mice and after anti-PDPN treatment. Mechanistically, a role for M1 activation in SAA pathogenesis may differ between the MIIG and anti-PDPN models; however, enhanced M2 activity correlates with protection in SAA.

Macrophages participate in immune-mediated thrombocytopenia (ITP), where increased platelet clearance drives thrombocytopenia and reduced platelet production. ${ }^{45} \mathrm{M} \phi$ clearance of platelets does not appear to drive thrombocytopenia in SAA, however, as clearance rates were similar in $\mathrm{M} \phi$-depleted and control mice. Our findings are consistent with and add to previous reports of $\mathrm{M} \phi$-dependent impairment of megakaryopoiesis and platelet production at steady state. ${ }^{46,47}$ Additionally, macrophage-colony stimulating factor M-CSF, a factor that increases $M \phi$ self-renewal, transiently causes thrombocytopenia. ${ }^{47}$ Our study builds upon these findings and identify M $\phi s$ as key sensors or target cells of IFN $\gamma$.

Acute inflammation increases CD41 ${ }^{\text {hi }} \mathrm{HSCs}$ or SL$\mathrm{MkPs}^{15}$ and we observed the emergence of a CD41 ${ }^{\mathrm{hi}} \mathrm{HSC}$ population in SAA that is accompanied by increased Mk numbers and platelet recovery when $M \phi s$ are depleted or unresponsive to IFN $\gamma$. While CD41 ${ }^{\mathrm{hi}}$ HSC-derived Mks may support sustained platelet production during SAA, another intriguing possibility is that Mk-lineage cells protect HSCs through a positive feedback loop. Mk-derived factors promote HSC quiescence and protect 
HSC from myeloablative injury. ${ }^{12,13}$ Therefore, CD $41^{\text {hi }}$ HSCs may represent more committed progenitors that augment HSC-protective niches during SAA. It is currently unclear if Mk preservation is necessary for or predictive of HSC rescue. However, our finding that HSC loss precedes Mk loss would argue in favor of a decline in megakaryopoiesis as a result of reduced CD41 $1^{\text {hi }} \mathrm{HSCs}$.

We identify a unique population of PDPN-expressing M $\phi$ s that restrict the HSC compartment and contribute to thrombocytopenia during SAA. PDPN regulates contractility and migration of lymphatic endothelial cells, FRCs, and tumor cells. ${ }^{48}$ Thus PDPN signaling in M $\phi s$ may influence hematopoiesis in a similar manner by regulating BM stiffness or migration within BM niches. Because increased or decreased matrix stiffness impairs proplatelet extension by Mks in vitro, it is possible that SAA-induced stromal stiffness restricts thrombopoiesis. ${ }^{45}$ PDPN expression is low and confined mainly to stromal cells in the BM at steady state and even upon radiation injury. PDPN is specifically increased on Mps in the BM during SAA, and anti-PDPN antibody specifically reduced $\mathrm{CD} 11 \mathrm{~b}^{\mathrm{lo} /} \mathrm{M} \phi \mathrm{s}$, but not $\mathrm{CD} 11 \mathrm{~b}^{+} \mathrm{M} \phi \mathrm{s}$, during SAA. Our data demonstrate a direct correlation between $\mathrm{CD} 11 \mathrm{~b}^{\mathrm{lo/}} \mathrm{M} \phi \mathrm{s}$ and SAA pathogenesis. Future studies to test the impact of PDPN expression on M $\phi$ migration and survival during SAA are warranted.

Podoplanin-expressing M $\phi$ s are increased in SAA, consistent with previous reports that PDPN is expressed on inflammatory $M \phi s$ in response to IFN $\gamma$ during infection. ${ }^{49}$ PDPN is a CLEC-2 ligand that triggers downstream signaling in CLEC2-expressing cells. However CLEC-2/PDPN ligation also elicits bidirectional signaling, eliciting RANTES production from PDPN-expressing cells. ${ }^{33,34}$ We noted increased RANTES in SAA, relative to radiation controls. Thus, our observation that PDPN blockade did not impact SAA-induced RANTES was somewhat surprising. However, our data and a previous report demonstrate that clone 8.1.1 does not interfere with CLEC-2 binding. ${ }^{34}$ Thus, PDPN blockade with clone 8.1.1 during SAA likely does not interfere with CLEC-2-driven RANTES production. Clone 8.1.1 may interfere with CLEC-2 binding, ${ }^{50}$ though very high concentrations of antibody were needed, and it is unlikely that this can be achieved in vivo. Our observations demonstrate protection independently of CLEC-2; however, future studies are necessary to test the involvement of the CLEC-2-PDPN axis and define the precise action of PDPN in the BM during SAA.

Interferon- $\gamma$ impacts SAA disease progression, yet neutralization of IFN $\gamma$ is not currently a treatment option during this disease. Our finding that IFN $\gamma$ maintains M $\phi s$ provides the rationale for targeting M $\phi s$ during $\mathrm{BM}$ failure. We reveal that M申s errantly express PDPN during SAA and antagonizing PDPN signaling rescues HSCs and enhances platelet output, thus revealing a novel circuit in the microenvironment during BM failure. Understanding the mechanisms whereby PDPN expression in Mфs regulates HSC function and platelet production may reveal novel treatment options for SAA.

\section{Acknowledgments}

We would like to acknowledge Kathleen Curran and Candace Ross at the New York State Department of Health Wadsworth Center (Albany, NY) for running CBCs on blood samples. We would like to thank Dr. Livingston Van De Water for helpful discussion.

\section{Funding}

This work was supported by R01 GM105949 to KCM, an Aplastic Anemia and MDS International Foundation grant to KCM, and BM160071 (DOD-BMFRP-IDA) to KCM.

\section{References}

1. Chen J. Animal models for acquired bone marrow failure syndromes. Clin Med Res. 2005;3(2):102-108

2. Scheinberg P, Chen J. Aplastic anemia: what have we learned from animal models and from the clinic. Semin Hematol. 2013; 50(2):156-164.

3. Young NS, Maciejewski J. The pathophysiology of acquired aplastic anemia. $N$ Engl Med. 1997;336(19):1365-1372.

4. Roderick JE, Gonzalez-Perez G, Kuksin CA, et al. Therapeutic targeting of NOTCH signaling ameliorates immune-mediated bone marrow failure of aplastic anemia. J Exp Med. 2013;210(7):1311-1329.

5. Bloom ML, Wolk AG, Simon-Stoos KL, Bard JS, Chen J, Young NS. A mouse model of lymphocyte infusion-induced bone marrow failure. Exp Hematol. 2004; 32(12):1163-1172.

6. Lu J, Basu A, Melenhorst JJ, Young NS, Brown KE. Analysis of T-cell repertoire in hepatitis-associated aplastic anemia. Blood. 2004;103(12):4588-4593.

7. Solomou EE, Keyvanfar K, Young NS. T-bet, a Th1 transcription factor, is up-regulated in $\mathrm{T}$ cells from patients with aplastic anemia. Blood. 2006;107(10):3983-3991.

8. Zoumbos NC, Ferris WO, Hsu SM, et al.
Analysis of lymphocyte subsets in patients with aplastic anaemia. Br J Haematol. 1984;58(1):95-105.

9. Chen J, Lipovsky K, Ellison FM, Calado RT, Young NS. Bystander destruction of hematopoietic progenitor and stem cells in a mouse model of infusion-induced bone marrow failure. Blood. 2004;104(6):16711678

10. Zoumbos NC, Gascon P, Djeu JY, Young NS. Interferon is a mediator of hematopoietic suppression in aplastic anemia in vitro and possibly in vivo. Proc Natl Acad Sci USA. 1985;82(1):188-192.

11. Townsley DM, Desmond R, Dunbar CE, Young NS. Pathophysiology and management of thrombocytopenia in bone marrow failure: possible clinical applications of TPO receptor agonists in aplastic anemia and myelodysplastic syndromes. Int J Hematol. 2013;98(1):48-55.

12. Zhao M, Perry JM, Marshall $\mathrm{H}$, et al. Megakaryocytes maintain homeostatic quiescence and promote post-injury regeneration of hematopoietic stem cells. Nat Med. 2014;20(11):1321-1326.

13. Bruns I, Lucas D, Pinho S, et al. Megakaryocytes regulate hematopoietic stem cell quiescence through CXCL4 secretion. Nat Med. 2014;20(11):1315-1320.

14. Avecilla ST, Hattori K, Heissig B, et al.
Chemokine-mediated interaction of hematopoietic progenitors with the bone marrow vascular niche is required for thrombopoiesis. Nat Med. 2004;10(1):6471.

15. Haas S, Hansson J, Klimmeck D, et al. Inflammation-Induced Emergency Megakaryopoiesis Driven by Hematopoietic Stem Cell-like Megakaryocyte Progenitors. Cell Stem Cell. 2015;17(4):422-434

16. Gekas C, Graf T. CD41 expression marks myeloid-biased adult hematopoietic stem cells and increases with age. Blood. 2013;121(22):4463-4472.

17. Chang MK, Raggatt LJ, Alexander KA, et al. Osteal tissue macrophages are intercalated throughout human and mouse bone lining tissues and regulate osteoblast function in vitro and in vivo. J Immunol. 2008;181(2): 1232-1244.

18. Chow A, Lucas D, Hidalgo A, et al. Bone marrow CD169+ macrophages promote the retention of hematopoietic stem and progenitor cells in the mesenchymal stem cell niche. J Exp Med. 2011;208(2):261-271.

19. Winkler IG, Sims NA, Pettit AR, et al. Bone marrow macrophages maintain hematopoietic stem cell (HSC) niches and their depletion mobilizes HSCs. Blood. 2010;116(23): 4815-4828. 


$$
\text { in }
$$

